\title{
Self-directed arm therapy at home after stroke with a sensor-based virtual reality training system
}

Frieder Wittmann ${ }^{1 \dagger}$, Jeremia P. Held ${ }^{2 *}$ D, Olivier Lambercy ${ }^{1}$, Michelle L. Starkey ${ }^{4}$, Armin Curt ${ }^{4}$, Raphael Höver $^{3}$, Roger Gassert ${ }^{1}\left(\mathbb{D}\right.$, Andreas R. Luft ${ }^{2,5}$ and Roman R. Gonzenbach ${ }^{2}$

\begin{abstract}
Background: The effect of rehabilitative training after stroke is dose-dependent. Out-patient rehabilitation training is often limited by transport logistics, financial resources and a lack of motivation/compliance. We studied the feasibility of an unsupervised arm therapy for self-directed rehabilitation therapy in patients' homes.
\end{abstract}

Methods: An open-label, single group study involving eleven patients with hemiparesis due to stroke ( $27 \pm 31$. 5 months post-stroke) was conducted. The patients trained with an inertial measurement unit (IMU)-based virtual reality system (ArmeoSenso) in their homes for six weeks. The self-selected dose of training with ArmeoSenso was the principal outcome measure whereas the Fugl-Meyer Assessment of the upper extremity (FMA-UE), the Wolf Motor Function Test (WMFT) and IMU-derived kinematic metrics were used to assess arm function, training intensity and trunk movement. Repeated measures one-way ANOVAs were used to assess differences in training duration and clinical scores over time.

Results: All subjects were able to use the system independently in their homes and no safety issues were reported. Patients trained on $26.5 \pm 11.5$ days out of 42 days for a duration of $137 \pm 120$ min per week. The weekly training duration did not change over the course of six weeks $(p=0.146)$. The arm function of these patients improved significantly by 4.1 points $(p=0.003)$ in the FMA-UE. Changes in the WMFT were not significant $(p=0.552)$. ArmeoSenso based metrics showed an improvement in arm function, a high number of reaching movements (387 per session), and minimal compensatory movements of the trunk while training.

Conclusions: Self-directed home therapy with an IMU-based home therapy system is safe and can provide a high dose of rehabilitative therapy. The assessments integrated into the system allow daily therapy monitoring, difficulty adaptation and detection of maladaptive motor patterns such as trunk movements during reaching.

Trial registration: Unique identifier: NCT02098135.

Keywords: Rehabilitation, Stroke, Feasibility, Arm, Virtual reality therapy, Video games

\footnotetext{
* Correspondence: jeremia.held@uzh.ch

${ }^{\dagger}$ Equal contributors

2Division of Vascular Neurology and Rehabilitation, Department of

Neurology, University Hospital Zurich, Zurich, Switzerland

Full list of author information is available at the end of the article
} 


\section{Background}

Functional outcome following stroke is positively correlated with the dose of the applied rehabilitative intervention [1]. Therefore, post-stroke therapy should be provided at a high intensity, a high frequency and over long periods of time [1, 2]. However, the delivery of intensive physical therapy requires extensive therapist support, is expensive, and is often limited by the low compliance and lack of motivation to perform rehabilitative training at the recommended frequency [3]. This can lead to functional deterioration, e.g., by learned nonuse of the affected limb [4].

Self-directed home therapy, supported by dedicated instrumented devices [5-7] or virtual reality gaming platforms [8-13], could help to increase the dose of rehabilitation at low cost without the need for direct supervision by a therapist. It is important that such home therapy adapts to changes in the subject's performance in order for it to remain challenging and motivating [8]. On the other hand, unsupervised rehabilitative training could lead to inefficient or harmful (i.e. maladaptive) movement sequences or pain, and could potentially worsen performance $[8,11,14]$. Home therapy should, therefore, include monitoring of movement quantity and quality. Several platforms dedicated to upper-extremity home rehabilitation have been proposed [6, 7, 15-17]. However, to the best of our knowledge only few were actually installed in the patients' homes for several weeks and tested for feasibility beyond case studies. These home studies always involved some external supervision, in the form of e.g. onsite visits $[16,17]$, tele-monitoring and adaption $[16,17]$ or telephone calls $[6,7]$, which might have affected compliance and motivation and thereby therapy dosage. However, such an approach requires manpower, which limits the affordability and scalability of home-based therapy. The feasibility and compliance of completely unsupervised upper-limb stroke therapy over the course of several weeks remains to be investigated.

In this paper we investigate the feasibility of self-directed home training with the custom-designed ArmeoSenso system [18], a virtual reality arm rehabilitation platform based on wearable inertial measurement units (IMU). In a clinical study involving eleven patients with hemiparesis of the arm due to stroke, we evaluated the ability to deliver therapy at a high dose through simple-to-use and entertaining, yet functionally relevant and adaptive rehabilitation games. Unsupervised, automated assessments integrated into each therapy session allowed monitoring of arm function, and detection of undesired compensatory movements.

\section{Methods}

\section{ArmeoSenso training system}

ArmeoSenso comprises a motion capture system based on wearable sensors in combination with an all-in-one touch screen computer (Inspiron 2330, Dell Inc., Fig. 1a). The therapy software provides a user-friendly graphical user interface, two therapy games, and two short automated assessments of arm function [18]. For real-time tracking of arm and trunk movements, the patient wears three IMUs (MotionPod 3, Movea Inc.) fixed to the lower and upper arm as well as the trunk (Fig. 1a). The IMUs measure acceleration, angular velocity and the magnetic field, all in three dimensions, and stream this data wirelessly to a receiver block, which is connected to the computer via USB and serves as a docking station to charge the sensors. A kinematic reconstruction estimates the orientation of the trunk, the upper- and the lower arm based on the Madgwick algorithm [19] and the corresponding joint positions are computed with forward kinematics [20]. This reconstruction serves as input for the assessments and therapeutic virtual reality games (Fig. 1b). By using the same virtual kinematic parameters for each patient, virtual sizes, e.g. distances or the size of targets, are normalized to the patient's body size. To discourage trunk inclination or rotation during pointing movements, the arm movements are computed and displayed relative to the trunk.

\section{Sequence of a training session}

A typical training session is illustrated in Fig. 1c. The patient uses the unaffected hand to touch a start button on the screen, which triggers visual instructions on how to remove the IMUs from the receiver block, don them on and perform a simple calibration procedure (i.e. guided start). For the calibration, the patient has to sit upright and hold the impaired arm in a horizontal position directed towards the screen for five seconds to determine the orientation of the IMUs on the patient's body. For calibration, the patient was allowed to use the unaffected arm for support.

Automated unsupervised assessments, conducted before every therapy session, evaluate arm function on two standardized tasks that remained identical throughout the therapy. The first was a pointing task which aims to evaluate the ability and time required for reaching a virtual target. The targets appear consecutively and in random order at nine pre-defined target positions located within the reachable workspace of a healthy individual. The patients are instructed to reach the target as quickly as possible and then stay on the target for two seconds. If a target is not reached within eight seconds, it disappears and a penalty time of eight seconds is taken instead. The number of targets reached and the mean time to reach the targets are reported. Joint angles are recorded to detect maladaptive compensatory movements such as excessive trunk inclination or trunk rotation during reaching. 

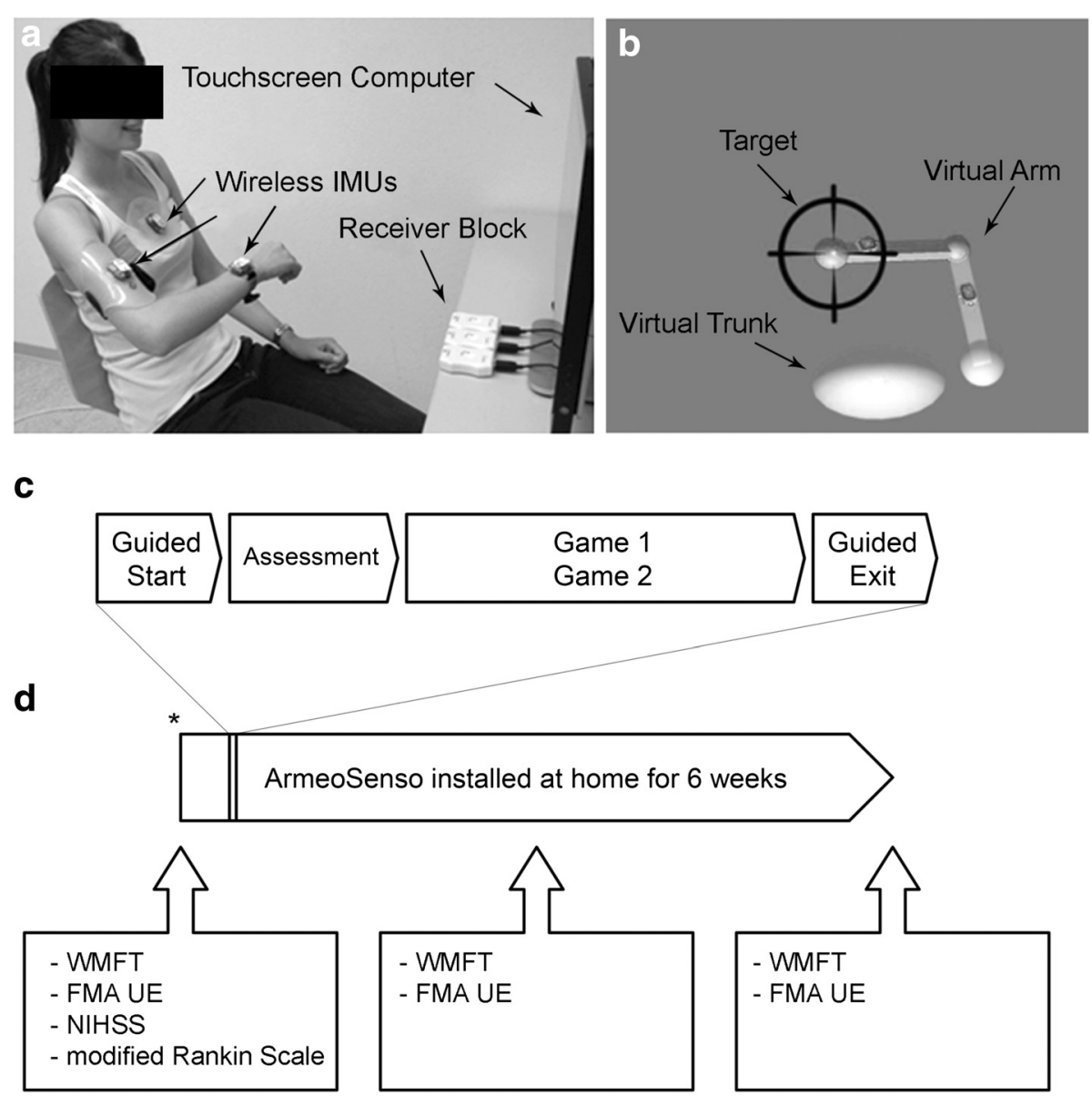

Fig. 1 System Overview and Study Outline. a: Photograph of a healthy subject using ArmeoSenso. b: Screenshot of the pointing task assessment: the virtual upper- and lower arm and the trunk are displayed. The arm points to a target. c: Sequence of a training session. Before each training session, two automated assessments are performed. $\mathbf{d}$ : Study outline: The ArmeoSenso system is installed in the patient's home for six weeks. The patients are assessed clinically before the start, after three weeks, and after six weeks of training. Abbreviations: WMFT: Wolf Motor Function Test; FMA-UE: Fugl-Meyer Assessment Upper Extremity; NIHSS: National Institute of Health Stroke Scale. *system installation and patient instruction by a therapist

The second assessment measured the two-dimensional workspace of the impaired arm in the transverse plane. Patients are instructed to actively reach out as far as possible with their impaired arm and to explore the entire arm workspace, similar to previous studies [21]. The attained workspace is displayed and computed as the number of squares of ten centimeters side length arranged in a transverse plane relative to the patient's trunk.

Therapy games: The aim of the therapy game 'Meteors', was to improve arm workspace and reaching velocity. In this game scenario, a virtual arm which matches the movement of the patient's arm is used to catch meteors that fall towards a planet. In contrast, the aim of the therapy game 'Slingshot' was to train arm coordination and to improve the precision of arm pointing and reaching movements. The patient holds a virtual slingshot to shoot stones at static or moving targets of variable size by pointing at the target with the slingshot and extending the elbow according to the target, which requires both precision and endurance. In both games, a performance-based (i.e. speed, number of targets reached, etc.) score is computed and used to dynamically adapt the difficulty of the game (e.g. meteors and targets move faster, or appear smaller etc.) in order to keep motivation and engagement high. The targets are placed within or at the border of the patient's 3D workspace, which is continuously estimated with a voxel-based model, to keep the challenge high, promote an increase in arm workspace, and prevent frustration [18].

\section{Study design}

The study was designed as an open-label, single group clinical trial to study the feasibility and safety of performing arm rehabilitation with the ArmeoSenso system in the patient's home without any supervision. Inclusion 
criteria were a minimum age of 18 years, hemiparesis of the arm due to cerebrovascular ischemia, the ability to lift the paretic arm against gravity, a minimal arm workspace of $20 \mathrm{~cm} \times 20 \mathrm{~cm}$ in the horizontal plane and absence of aphasia, depression, dementia and hemianopia. ArmeoSenso was installed on a table, and instructions for proper usage were given by a trained physiotherapist to the patient, prior to the start of the study. No modification of the patient's house was required. Patients were asked to use the system as often as possible over a period of six weeks. They decided by themselves about the training duration and frequency and could start or stop a therapy session at any time. The patients' usual therapy continued and was not altered during the study. A structured patient interview was conducted at the end of the trial. The study followed GCP-guidelines and was approved by the local Cantonal ethics committee Zurich (KEK-ZH: 2013-0182) and the Swissmedic (2013-MD0019). All subjects gave written informed consent in accordance with the declaration of Helsinki.

\section{Outcome measures}

The primary safety outcome was any adverse event related to the system that occurred during the study period. The primary outcome of the study was the duration of training per week with ArmeoSenso. This was used as an indicator of therapy acceptance and feasibility of unsupervised therapy. As a measure of motivation, we investigated whether system usage changed over time. We report the average training duration for every training week, the training duration per session (equal to the minutes of playing games per ArmeoSenso session) and the training frequency (equal to the number of days of ArmeoSenso usage). The sum of both the number of meteors caught (Meteors game) and the number of targets hit (Slingshot game) was used as a measure of training intensity. To assess the efficiency of training, we quantified the training duration in relation to the overall time spent with the system, which includes the time for automated assessments and for system setup.

To investigate whether patients compensated for their arm impairment by moving their trunk, we analyzed trunk rotation and inclination during successful pointing movements in the pointing task assessment for one target. Trunk rotation and inclination were recorded at onset (initiation) of the movement and once the hand reached the target (final). The respective absolute difference between initial and final trunk orientation was treated as the patient's trunk compensation. The subtraction also serves to remove bias, e.g. due to sensor misalignment or magnetic field disturbances [22, 23], while the short duration of $8 \mathrm{~s}$ or less should minimize effects of orientation drift [24], e.g. due to gyroscope bias. As a control, patients performed the same task with their unaffected arm 10 consecutive times within one session at the end of the home trial.

Arm function was assessed clinically using the FuglMeyer Assessment - Upper Extremity (FMA-UE) [25] and the Wolf Motor Function Test (WMFT) [26] at 3 time points (see Fig. 1d) and with ArmeoSenso-based automated assessments, as described above.

\section{Statistical methods}

Descriptive statistics are reported as mean \pm standard deviation of the mean, and where relevant with (min, $\max )$. All outcomes were inspected for normal distribution using the Kolmogorov-Smirnov test, prior to selection of appropriate statistical tests. The two tailed Mann-Whitney test was used to compare the average weekly training duration in patients with severe impairment of arm function against patients with moderate to mild impairment. A one-way repeated measures ANOVA was used to assess differences over time in training duration, clinical scores and automated assessments, in case of normally distributed data. Otherwise, the non-parametric Friedman test was used. Correlation analysis was used to examine the relationship between clinical assessments and ArmeoSenso-based assessments. Results were considered significant at $p<0.05$.

\section{Patient characteristics}

Eleven patients were recruited in the University Hospital Zurich (for details see Table 1). In parallel to the study, all patients except for one received physical therapy, on average 3.9 sessions/week, corresponding to approximately $155 \mathrm{~min} /$ week (estimated duration of $40 \mathrm{~min}$ per therapy session). Only one patient reported that he had no prior experience in using computers, and 8 out of 11 patients reported that they had never played computer games before.

Table 1 Baseline characteristics

\begin{tabular}{ll}
\hline & Mean $\pm \mathrm{SD}^{\mathrm{a}}(\min , \max )$ \\
\hline $\mathrm{N}$ & 11 \\
Male & 5 \\
Right side affected & 8 \\
Age, $y$ & $60 \pm 11.5(\min 42, \max 79)$ \\
Months post stroke & $27 \pm 31.5(\min 4, \max 118)$ \\
NIHSS $^{b}$ & $3.3 \pm 1.2(\min 1, \max 5)$ \\
mRS $^{c}$ & $1.9 \pm 0.1(\min 1, \max 3)$ \\
FMA-UE $^{c}$ & $35.1 \pm 19.9(\min 11, \max 60)$ \\
WMFT $^{d}$ & $52 \pm 39(\min 16, \max 70)$ \\
\hline
\end{tabular}

${ }^{\mathrm{a} S t a n d a r d}$ deviation

${ }^{\mathrm{b}}$ National Institutes of Health Stroke Scale (0-42 points)

'modified Rankin Scale (0-6 points)

${ }^{\mathrm{C}}$ Fugl-Meyer Assessment - Upper Extremity (0-66 points)

dWolf Motor Function Test (0-75 points) 


\section{Results}

Safety and system usage

All subjects were able to use the system without supervision at their homes and there were no patient-reported adverse events. On average, patients used the system on $26.5 \pm 11.5$ days ( $\min 8, \max 41$ ) out of 42 days (Fig. 2c), corresponding to 4.4 days with training per week. The average training duration per week was $137 \pm 120$ min (min 15, max 357). The weekly training duration did not change over the course of six weeks (one-way repeated measures ANOVA: $p=0.146, \mathrm{~F}=1.912$, Fig. 2a). According to the patient interviews, $8 / 11$ patients would have liked to continue training with the system and the perceived therapy efficacy was high, with $8 / 11$ patients stating that the trial improved their arm function. Further, $9 / 11$ of patients found the system to be motivating. The two patients who replied negatively were also within the group of those $3 / 11$ patients not stating a desire to continue training with the system. Further, these three patients had a significantly (Wilcoxon rank-sum test, $p=0.049$ ) lower initial FMA-UE score $(16 \pm 7.8)$ compared to the other eight patients (FMA-UE $43 \pm 18.3$ ). These patients also trained less (85 $\mathrm{min} / \mathrm{w}$ versus $177 \mathrm{~min} / \mathrm{w}$ ), but the difference was not significant (Wilcoxon rank-sum test, $\mathrm{p}=0.38$ ).

The average training (gaming) duration per session was $30 \pm 16 \min (\min 11, \max 56)$ (Fig. 2b). The average number of successful arm movements during gaming with Meteors and Slingshot, a measure for training intensity, was $387 \pm 522$ movements per session (min 40, $\max$ 1486). Patients with severe impairment of arm function (FMA-UE $\leq 20, N=4$ ) used ArmeoSenso significantly less $(42 \pm 42 \mathrm{~min} /$ week $)$ than those with
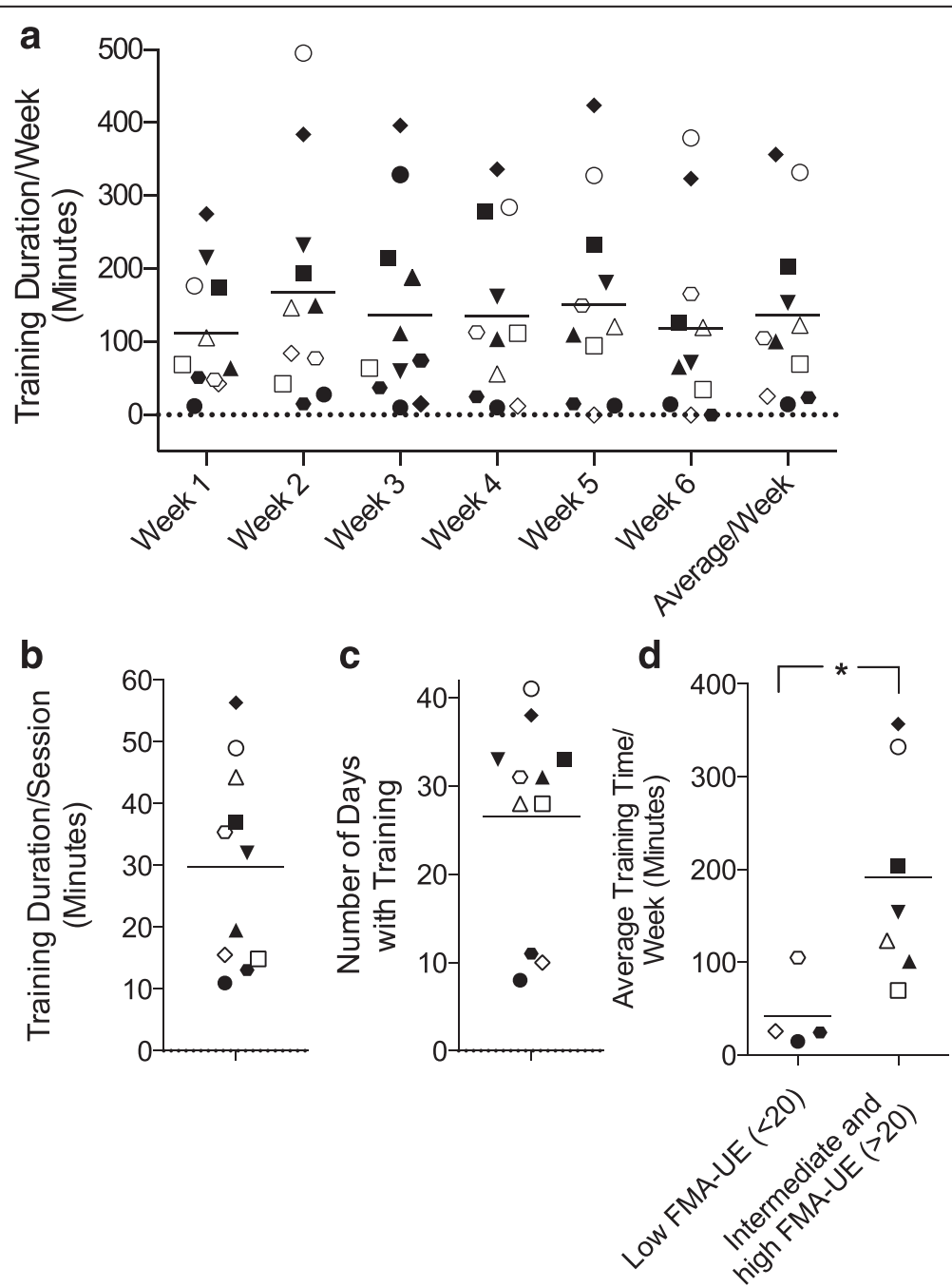

Fig. 2 System Usage: a-d: Each symbol represents one patient. a: Weekly training duration for weeks 1-6 and average weekly training duration for each patient. b: Training duration per session. c: Number of days with training. Horizontal lines indicate averages. d: Average weekly training duration in patients with low (<20 points) Fugl-Meyer Assessment Upper Extremity (FMA-UE) and intermediate to high ( $>20$ points) FMA-UE score. * indicates significant differences in usage 
moderate and mild arm impairment (FMA-UE $>20, N=$ 7, $191 \pm 113 \mathrm{~min} /$ week, $p=0.024$, Fig. $2 \mathrm{~d}$ ).

The average setup duration per therapy session was $4 \pm$ $2 \mathrm{~min}$. The combined average time to complete all assessments for a therapy session was $4 \pm 1 \mathrm{~min}$. On average, patients spent $79 \%$ of a therapy session with actual training, i.e. playing either the Meteors or Slingshot therapy game.

\section{Changes in arm function}

Patients showed a significant improvement in the FMA-UE from $35.1 \pm 19.9$ points to $39.2 \pm 17.9$ points after 6 weeks, which represents an average improvement of $4.1 \pm 2.5$ points (one way repeated measures ANOVA: $p=0.003$, $F=8.701$, Fig. 3a). The changes seen in the WMFT were small and not significant (improvement of +1.2 points after six weeks, Friedman-test: $p=0.552$ ).

The automated assessments performed at each training session were plotted as two-weekly averages for comparison to clinical scores. The workspace of the affected arm in the transverse plane, as documented by the automated workspace assessment, improved significantly by $31 \%$ between the first two weeks $(31.5 \pm 20.8$ squares $)$ and the last two weeks (40.8 \pm 28 squares; one way ANOVA: $p=0.008, \mathrm{~F}=$ 9.280, Fig. $3 \mathrm{~b}$ ). In the pointing task, the number of targets (out of 9) reached within $8 \mathrm{~s}$ improved significantly from $4.4 \pm 2.8$ in the first two weeks to $5.9 \pm 3.1$ in the last two weeks (Friedman-test: $p<0.001, \mathrm{~F}=13.780$, data not shown). The average time to reach the targets decreased significantly by $19 \%$, from $5.4 \pm 1.6 \mathrm{~s}$ in the first two weeks to $4.5 \pm 2.2 \mathrm{~s}$ in the last two weeks (one-way ANOVA: $p=$ $0.005, \mathrm{~F}=7.17$, Fig. 3c).

The FMA-UE scores correlated significantly with all three metrics of the automated assessments (number of workspace voxels $\mathrm{r}=0.91, p<0.001$, number of reached targets $r=0.96, p<0.001$, time to reach target $r=0.92, p<0.001$, the latter is shown in Fig. $3 d$ ).
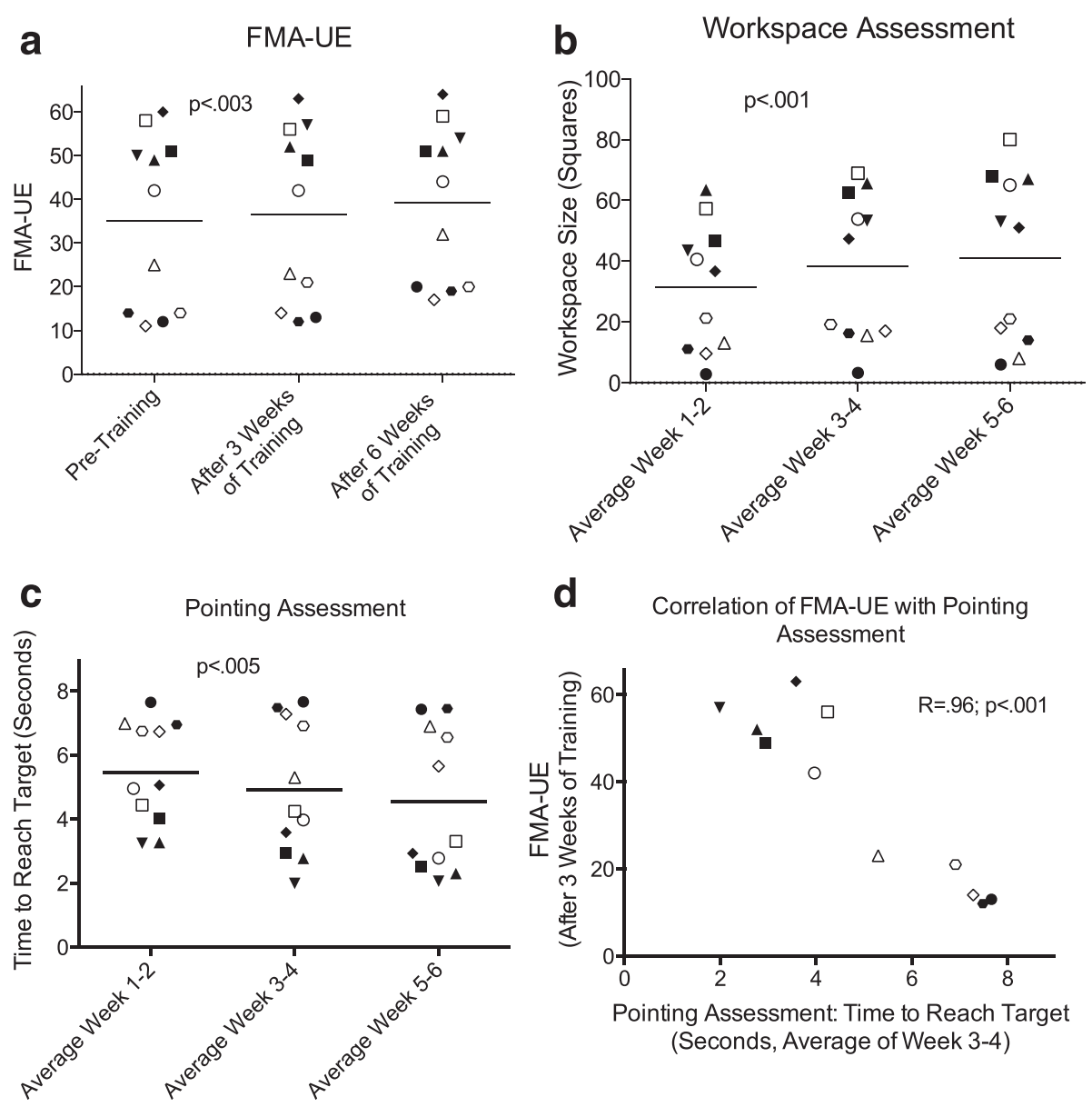

Fig. 3 Arm Function Assessments: a-d: Each symbol represents one patient. a-c: Horizontal bar = average. a: Fugl-Meyer Assessment Upper Extremity (FMA-UE) shows significant improvement after six weeks of therapy. b-d: ArmeoSenso-based Assessments. In one instance, a patient did not use the system during a block of two weeks. Here, the previous value was carried forward. $\mathbf{b}$ : Arm Workspace Assessment. The workspace is reported as squares, i.e. relative units for the covered workspace and shows significant improvement after six weeks. c: Pointing Task Assessment. The average time to reach targets improves significantly. $\mathbf{d}$ : Significant correlation between clinical assessment (Fugl-Meyer assessment after 3 weeks of training) and ArmeoSenso assessment (time to reach target, average of training week 3-4, c) 
The therapy dose (i.e. total training duration with ArmeoSenso) did not correlate with the changes over six weeks in the clinical assessments $(\mathrm{r}=-0.3, p=0.370$ for the FMA-UE, and $r=-0.083, p=0.809$ for the WMFT, data not shown).

\section{Kinematic analysis}

Trunk angle analysis during the pointing assessment shows that patients moved their trunk significantly more when reaching with their impaired arm compared to reaching with their unaffected arm (Fig. 4). These trunk movements occurred with a higher variability in the impaired side, as demonstrated by high standard deviations. The average absolute trunk rotation did not change significantly between the first and the last two weeks (one-way ANOVA, $\mathrm{p}=0.531, \mathrm{~F}=0.415$ ) but was significantly higher when compared to reaching movements with the unaffected arm (one-way ANOVA, $p=$ $0.030, F=5.859)$. The same was observed for the average trunk inclination (for target 6), which did not change significantly between the first and the last two weeks (one-way ANOVA, $p=0.208, \mathrm{~F}=1.757$ ) but was significantly higher compared to the sessions with the unaffected arm (one-way ANOVA, $p<0.001, \mathrm{~F}=24.968$ ).
An example of the high inter-session variability of trunk angles during all training sessions for one patient (and the same target) is shown in Fig. $4 \mathrm{~b}$ and d.

\section{Discussion}

This paper presents results of a feasibility study using ArmeoSenso, a novel, wearable sensor-based home therapy system with rehabilitative games for arm training and automated IMU-based assessments of arm function. During a six-week intervention, all stroke patients $(N=11)$ were able to train with ArmeoSenso at home without therapist supervision and with no side effects reported, demonstrating that unsupervised selfdirected home therapy using a sensor-based virtual therapy platform is feasible and safe. As automated rehabilitation systems carry the risk of being unsuitable for stroke patients due to their complexity, we placed a high priority on developing a system that was easy to use, with therapy exercises that involved intuitive and meaningful, yet challenging movement tasks [27]. The fact that elderly patients $(6 / 11$ were aged $>60$ years) and patients without gaming experience $(8 / 11)$ were able to successfully use the system supports its broad applicability. However, patients with severe impairments of arm

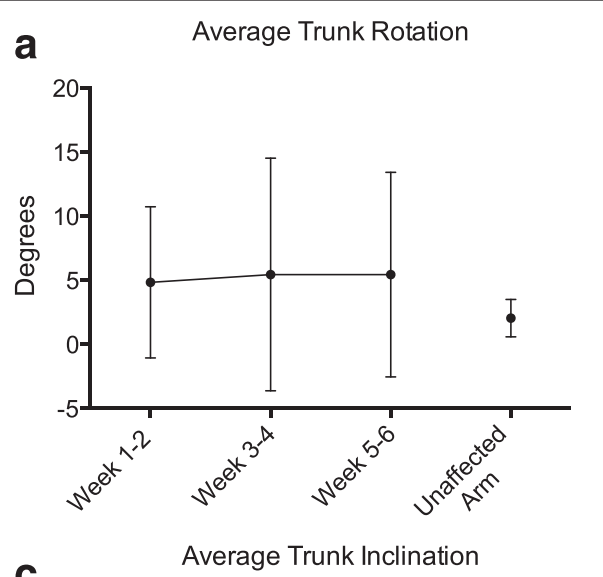

b Patient 4 - Trunk Rotation

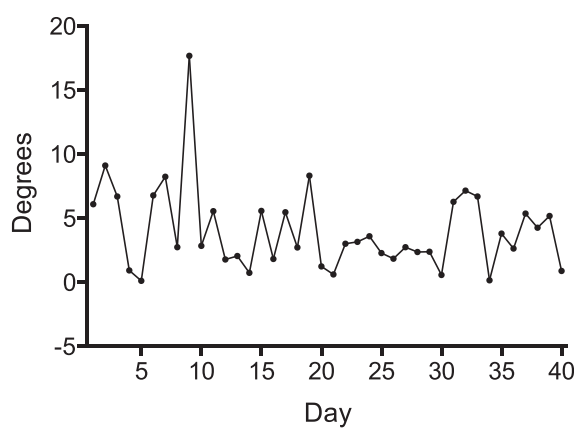

d Patient 4 - Trunk Inclination
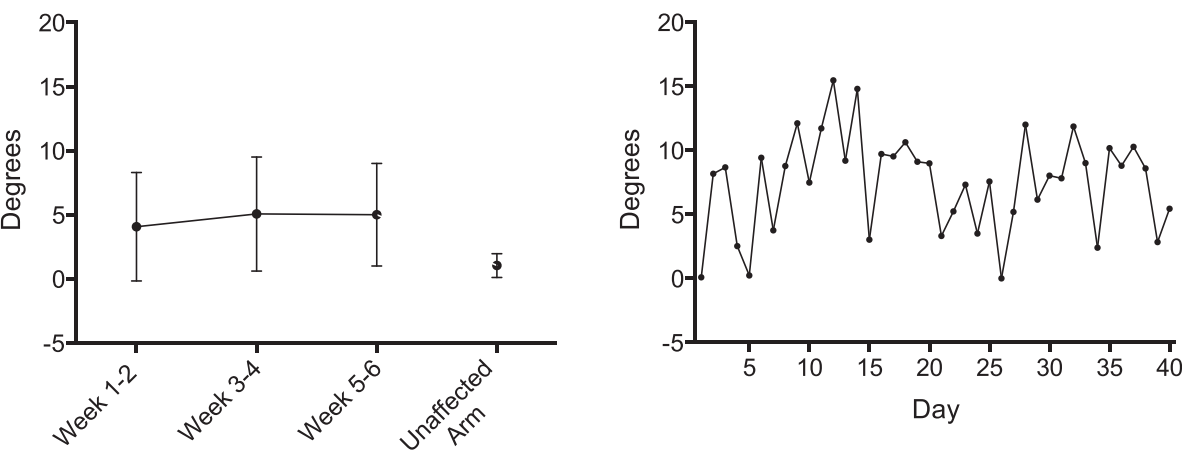

Fig. 4 Trunk Movement during Pointing. Trunk rotation $(\mathbf{a}, \mathbf{b})$ and inclination (c, d) (two-weekly average) during pointing movements in the pointing task assessment for one specific target. For comparison, the values of 10 pointing movements performed with the unaffected limb are plotted $(N=8) . \mathbf{b}+\mathbf{d}$ : To demonstrate the high inter-session variability of trunk rotation and inclination during pointing movements, a complete dataset of one patient (impaired side) is plotted for the same target. Error bars: standard deviation 
function used the system less than those with moderate or mild impairments, suggesting that targeted training systems for this group should be developed, e.g. by addition of gravity support.

The therapy dose of $137 \mathrm{~min}$ per week (min/week) on average, with training sessions on 4.4 days per week, is promising. Despite the lack of any external therapy supervision after the initial setup day, this result compares favorably to other studies on unsupervised therapy in stroke, where doses of $105 \mathrm{~min} /$ week were achieved with the "Supervised Care \& Rehabilitation Involving Personal Telerobotics" (SCRIPT) hand orthosis [16, 28], $85 \mathrm{~min} /$ week with the "home-based Computer Assisted Arm Rehabilitation" (hCAAR) actuated joystick [7] or $31 \mathrm{~min} /$ week with the Virtual Glove upper-limb rehabilitation system [17]. Higher training doses of $214 \mathrm{~min} /$ week were achieved with the "Elinor" home therapy system [13], but mandatory weekly hospital visits might have influenced patient compliance. The average training intensity, which was 387 successful reaching and pointing movements per session, is in the range of another study with self-directed home therapy for subacute stroke patients, where 383 exercise repetitions per session were reported [29], and is much higher than the relatively low intensity typically observed in standard rehabilitation sessions for the upper limb (32 functional upper extremity movements per session [30]). The observed training duration did not decline during the six week intervention, indicating that the motivation to train with ArmeoSenso remained high. Overall system usage and the reported desire to continue training after completion of the study protocol suggest that the therapy could even be applied over longer periods. Training efficiency was also high with patients spending almost $80 \%$ of the time using the ArmeoSenso system with actual rehabilitation training. This compares favorably to training times in routine outpatient therapy [31]. Such high training efficiency might lower the threshold to start a therapy session and thus increase the therapy dose within the available time. The system's permanent availability throughout the day in the subjects' home without the restriction of clinical schedules is an important advantage over tele-rehabilitation approaches [32, 33]. It would be interesting to know how many patients declined to participate and the reasons they give for this. However, this was not documented in this feasibility study. In two cases, there was insufficient space to set up the system in the patient's homes, which illustrated the fact that size and footprint is an important design criterion for a home-based rehabilitation system.

In the unsupervised setting used here, research therapist did not see the patient over the course of the therapy. Direct monitoring of performance and progress and external intervention was therefore not possible. This motivated the development of short assessment modules that patients performed on a daily basis. To the best of our knowledge, unsupervised, automated assessments that accompany each training session have not been realized until now. The high correlation found between the automated assessments and clinical assessment scales in arm function is a first step towards confirming their validity. In the future, such unsupervised, automated assessments could alert therapists remotely, e.g. via the Internet, about stagnating or declining performance during home training sessions. Therapy games which do not take into account a patient's individual impairment, as with commercial entertainment systems designed for healthy users, are likely to frustrate patients, potentially jeopardizing motivation and compliance. ArmeoSenso therapy games constantly adapt their difficulty and intensity according to the subjects' performance, and place targets within or at the border of the reachable workspace [18] to maximize engagement and motivation of the subject.

An important function of a therapist is also to monitor and, if needed, correct the patient's posture and movements in order to prevent the development of pain or maladaptive motor patterns, such as excessive compensatory trunk movements (inclination and rotation) or excessive shoulder abduction during arm reaching [34]. With systems that do not track joint angles (e.g. Nintendo Wii) or commercial games that are not designed for rehabilitation purposes [11,35], development of such patterns may go unnoticed. Systems based on the use of cameras (e.g. Kinect [12, 35-37]) or IMUs that reconstruct body posture offer the possibility to detect compensatory movements [38]. The reconstruction algorithm implemented in ArmeoSenso attempted to minimize trunk inclination and rotation by directly suppressing their effects in the virtual environment, i.e. only arm movements relative to the trunk are depicted and used as input for the games and assessments. Despite this effort, patients typically exhibited significantly higher trunk inclination and trunk rotation during reaching movements with their impaired arm than with the unimpaired arm [39]. Nevertheless, trunk movements remained small in most patients, with an average of less than five degrees of trunk inclination or rotation. The extent of trunk movement was highly variable (interpatient and inter-session), and there was no significant trend over time that would suggest either an increase or a reduction of compensation with the trunk during reaching movements. Providing auditory instructions when excessive trunk movements are detected, simulating the presence of a virtual therapist [38], or using negative visual cues within the therapy game [40], might help to prevent compensatory trunk movements. 
The mean gain in FMA-UE was 4.1 points, which is not regarded as clinically relevant, but five out of eleven patients showed a clinically relevant improvement of more than 4.25 points [41]. This is comparable to findings for high-intensity therapy in chronic stroke patients [42]. This improvement was not reflected in the WMFT, which improved marginally by 1.2 points. This is likely due to the lack of hand training by ArmeoSenso; hand function is important for performing the WMFT. The improvement of arm function could be explained by the self-directed training with ArmeoSenso or by the standard rehabilitation therapy that most patients received during the study in addition to the experimental training (155 min per week on average).

\section{Conclusion}

This paper presents the design and feasibility of ArmeoSenso, a wearable sensor-based home therapy system for self-directed rehabilitative arm training after stroke. Our results demonstrate that this home therapy is safe and can provide rehabilitative training in a high dose. The integrated assessments allow daily therapy monitoring, difficulty adaptation and detection of maladaptive motor patterns such as trunk movements during reaching. Clinical effectiveness of ArmeoSenso needs to be investigated in a larger randomized controlled trial.

\section{Abbreviations}

FMA-UE, Fugl-Meyer assessment of the upper extremity; GCP, Good Clinical Practice; IMU, Inertial Measurement Unit; WMFT, Wolf Motor Function Test

\section{Acknowledgments}

The authors would like to thank Mark van Raai, Dr. Kei Hoshi, Dr. Peter Schenk, Dr. Melissa Lawrence and Dr. Kaspar Leuenberger for their help in the implementation of the ArmeoSenso software, Irene Christen, Lydia Fischer, Werner Popp and Urs Albisser for their support with the study, Dr. Corinne Nicoletti for setup pictures, as well as all patients who participated in the study.

\section{Funding}

This work was supported by the Swiss Commission for Technology and Innovation (CTI Grant 13612.1) in collaboration with Hocoma AG, the Clinical Research Priority Program (CRPP) NeuroRehab of the University of Zurich, the ETH Foundation (ETH Research Grant ET-17 13-2) and the P\&K Pühringer Foundation.

\section{Authors' contributions}

RRG, OL, FW, JPH and RG drafted the manuscript. FW, JPH, OL, MLS, AC, RH, $R G, A R L$ and RRG participated in the study design and definition of requirements. FW and JPH implemented the therapy system and performed the data analysis. JPH, RRG and FW recruited patients, ran the clinical study, and carried out the statistical analysis. All authors read and approved the final manuscript.

\section{Availability of data and supporting materials}

The data supporting the conclusions of this article are included within the article.

\section{Competing interests}

Raphael Höver is employed by Hocoma AG (Volketswil, Switzerland), a company that manufactures and markets rehabilitation technology devices. Furthermore, Andreas R. Luft is a scientific advisor to Hocoma AG (Volketswil, Switzerland). The remaining authors have no conflict of interest in the submission of this manuscript.

\section{Consent for publication}

The individual in Fig. 1a consented to the publication of the photograph.

\section{Ethics approval and consent to participate}

The study followed GCP-guidelines and was approved by the local Cantonal ethics committee Zurich (KEK-ZH: 2013-0182) and Swissmedic (2013MD-0019). All subjects gave written informed consent in accordance with the declaration of Helsinki.

\section{Author details}

${ }^{1}$ Rehabilitation Engineering Laboratory, Department of Health Sciences and Technology, ETH Zurich, Switzerland. 'Division of Vascular Neurology and Rehabilitation, Department of Neurology, University Hospital Zurich, Zurich, Switzerland. ${ }^{3}$ Hocoma AG, Volketswil, Switzerland. ${ }^{4}$ Spinal Cord Injury Center, Balgrist University Hospital, Zurich, Switzerland. ${ }^{5}$ Cereneo Center for Neurology and Rehabilitation, Vitznau, Switzerland.

Received: 23 February 2016 Accepted: 26 July 2016

Published online: 11 August 2016

\section{References}

1. Kwakkel G. Impact of intensity of practice after stroke: issues for consideration. Disabil Rehabil. 2006;28(13-14):823-30.

2. Lohse KR, Lang CE, Boyd LA. Is more better? Using metadata to explore dose-response relationships in stroke rehabilitation. Stroke. 2014;45(7):2053-8.

3. Lenze EJ, Munin MC, Quear T, Dew MA, Rogers JC, Begley AE, Reynolds CF. Significance of poor patient participation in physical and occupational therapy for functional outcome and length of stay. Arch Phys Med Rehabil. 2004;85(10):1599-601.

4. Taub E. Movement in nonhuman primates deprived of somatosensory feedback. Exerc Sport Sci Rev. 1976;4:335-74.

5. Kohler F, Schmitz-Rode T, Disselhorst-Klug C. Introducing a feedback training system for guided home rehabilitation. J Neuroeng Rehabil. 2010;7:2.

6. Zondervan DK, Augsburger R, Bodenhoefer B, Friedman N, Reinkensmeyer DJ, Cramer SC. Machine-based, self-guided home therapy for individuals with severe arm impairment after stroke: a randomized controlled trial. Neurorehabil Neural Repair. 2015;29(5):395-406.

7. Sivan M, Gallagher J, Makower S, Keeling D, Bhakta B, O'Connor RJ, Levesley M. Home-based computer assisted arm rehabilitation (hCAAR) robotic device for upper limb exercise after stroke: results of a feasibility study in home setting. J Neuroeng Rehabil. 2014;11(1):163.

8. Borghese NA, Pirovano M, Lanzi PL, Wuest S, de Bruin ED. Computational intelligence and game design for effective at-home stroke rehabilitation. Games health J. 2013;2(2):81-8.

9. Cameirao MDS, Badia SBI, Duarte E, Verschure PFMJ. Virtual reality based rehabilitation speeds up functional recovery of the upper extremities after stroke: a randomized controlled pilot study in the acute phase of stroke using the rehabilitation gaming system. Restor Neurol Neuros. 2011;29(5):287-98

10. Saposnik G, Robert T, Mamdani M, Cheung D, Thorpe KE, Mcllroy B, Willems J, Hall J, Cohen LG, Bayley M. Effectiveness of virtual reality using wii gaming technology in stroke rehabilitation (EVREST): a randomized clinical trial and proof of principle. Stroke. 2010;41(7):E473-3.

11. Prosperini L, Fortuna D, Gianni C, Leonardi L, Marchetti MR, Pozzilli C. Home-based balance training using the Wii balance board: a randomized, crossover pilot study in multiple sclerosis. Neurorehabil Neural Repair. 2013;27(6):516-25

12. Webster D, Celik O. Systematic review of Kinect applications in elderly care and stroke rehabilitation. J Neuroeng Rehabil. 2014;11:108.

13. Slijper A, Svensson KE, Backlund P, Engstrom H, Sunnerhagen KS. Computer game-based upper extremity training in the home environment in stroke persons: a single subject design. J Neuroeng Rehabil. 2014;11:35.

14. Liebermann DG, Berman S, Weiss PL, Levin MF. Kinematics of reaching movements in a 2-D virtual environment in adults with and without stroke. leee T Neur Sys Reh. 2012;20(6):778-87.

15. Kim GJ, Rivera L, Stein J. Combined clinic-home approach for upper limb robotic therapy after stroke: a pilot study. Arch Phys Med Rehabil. 2015;96(12):2243-8.

16. Nijenhuis SM, Prange GB, Amirabdollahian F, Sale P, Infarinato F, Nasr N, Mountain G, Hermens HJ, Stienen AHA, Buurke JH, et al. Feasibility study into self-administered training at home using an arm and hand device with 
motivational gaming environment in chronic stroke. J Neuroeng Rehabil. 2015;12.

17. Standen PJ, Threapleton K, Connell L, Richardson A, Brown DJ, Battersby S, Sutton CJ, Platts F. Patients' use of a home-based virtual reality system to provide rehabilitation of the upper limb following stroke. Phys Ther. 2015;95(3):350-9.

18. Wittmann F, Lambercy O, Gonzenbach RR, van Raai MA, Hover R, Held J, Starkey ML, Curt A, Luft A, Gassert R. Assessment-driven arm therapy at home using an IMU-based virtual reality system. Singapore: IEEE International Conference on Rehabilitation Robotics (ICORR). 2015;707-712.

19. Madgwick SOH, Harrison AJL, Vaidyanathan R. Estimation of IMU and MARG orientation using a gradient descent algorithm. Zurich:IEEE International Conference on Rehabilitation Robotics (ICORR). 2011: 1-7.

20. Craig JJ. Introduction to Robotics: Mechanics and Control: Vol. 3,. Upper Saddle River: Pearson/Prentice Hall; 2005.

21. Sukal TM, Ellis MD, Dewald JP. Shoulder abduction-induced reductions in reaching work area following hemiparetic stroke: neuroscientific implications. Exp Brain Res. 2007;183(2):215-23.

22. Roetenberg D, Luinge HJ, Baten CT, Veltink PH. Compensation of magnetic disturbances improves inertial and magnetic sensing of human body segment orientation. IEEE Trans Neural Syst Rehabil Eng. 2005;13(3):395-405.

23. Roetenberg $D$, Baten $C T$, Veltink PH. Estimating body segment orientation by applying inertial and magnetic sensing near ferromagnetic materials. IEEE Trans Neural Syst Rehabil Eng. 2007;15(3):469-71.

24. LaValle SM, Yershova A, Katsev M, Antonov M. Head tracking for the Oculus Rift. Hong Kong: IEEE International Conference on Robotics and Automation (ICRA). 2014;187-194.

25. Fugl-Meyer AR, Jaasko L, Leyman I, Olsson S, Steglind S. The post-stroke hemiplegic patient. 1. A method for evaluation of physical performance. Scand J Rehabil Med. 1975;7(1):13-31.

26. Wolf SL1, Catlin PA, Ellis M, Archer AL, Morgan B, Piacentino A. Assessing Wolf motor function test as outcome measure for research in patients after stroke. Stroke. 2001;32(7):1635-9.

27. Burke JW, McNeill MDJ, Charles DK, Morrow PJ, Crosbie JH, McDonough SM. Optimising engagement for stroke rehabilitation using serious games. Visual Comput. 2009:25(12):1085-99.

28. Amirabdollahian F, Ates S, Basteris A, Cesario A, Buurke J, Hermens H, Hofs D, Johansson E, Mountain G, Nasr N. Design, development and deployment of a hand/wrist exoskeleton for home-based rehabilitation after strokeSCRIPT project. Robotica. 2014;32(08):1331-46.

29. Zondervan DK, Augsburger R, Bodenhoefer B, Friedman N, Reinkensmeyer DJ, Cramer SC. Machine-based, self-guided home therapy for individuals with severe arm impairment after stroke: a randomized controlled trial. Neurorehabil Neural Repair. 2014.

30. Lang CE, Macdonald JR, Reisman DS, Boyd L, Jacobson Kimberley T, Schindler-Ivens SM, Hornby TG, Ross SA, Scheets PL. Observation of amounts of movement practice provided during stroke rehabilitation. Arch Phys Med Rehabil. 2009;90(10):1692-8.

31. De Wit L, Putman K, Dejaeger E, Baert I, Berman P, Bogaerts K, Brinkmann N, Connell L, Feys $H$, Jenni W, et al. Use of time by stroke patients: a comparison of four European rehabilitation centers. Stroke. 2005;36(9):1977-83.

32. Laver KE, Schoene D, Crotty M, George S, Lannin NA, Sherrington C. Telerehabilitation services for stroke. Cochrane Database Syst Rev. 2013;12, CD010255.

33. Johansson T, Wild C. Telerehabilitation in stroke care-a systematic review. J Telemed Telecare. 2011;17(1):1-6.

34. Cirstea MC, Levin MF. Compensatory strategies for reaching in stroke. Brain. 2000;123(Pt 5):940-53.

35. Sin $\mathrm{H}$, Lee G. Additional virtual reality training using Xbox Kinect in stroke survivors with hemiplegia. Am J Phys Med Rehabil. 2013;92(10):871-80.

36. Gama AD, Fallavollita P, Teichrieb V, Navab N. Motor rehabilitation using kinect: a systematic review. Games Health J. 2015;4(2):123-35.

37. Taati B, Wang R, Huq R, Snoek J, Mihailidis A. Vision-based posture assessment to detect and categorize compensation during robotic rehabilitation therapy. P leee Ras-Embs Int. 2012;1607-1613.

38. Johnson MJ, Shakya Y, Strachota E, Ahamed SI. Low-cost monitoring of patients during unsupervised robot/computer assisted motivating stroke rehabilitation. Biomed Tech. 2011;56(1):5-9.

39. Mandon L, Boudarham J, Robertson J, Bensmail D, Roche N, Roby-Brami A. Faster reaching in chronic spastic stroke patients comes at the expense of arm-trunk coordination. Neurorehabil Neural Repair. 2015.
40. Alankus G, Kelleher C. Reducing compensatory motions in motion-based video games for stroke rehabilitation. Hum-Comput Interact. 2015;30(3-4):232-62.

41. Page SJ, Fulk GD, Boyne P. Clinically important differences for the upperextremity Fugl-Meyer Scale in people with minimal to moderate impairment due to chronic stroke. Phys Ther. 2012;92(6):791-8.

42. Wu X, Guarino P, Lo AC, Peduzzi P, Wininger M. Long-term Effectiveness of Intensive Therapy in Chronic Stroke. Neurorehabil Neural Repair. 2016;30(6): 583-90

\section{Submit your next manuscript to BioMed Central and we will help you at every step:}

- We accept pre-submission inquiries

- Our selector tool helps you to find the most relevant journal

- We provide round the clock customer support

- Convenient online submission

- Thorough peer review

- Inclusion in PubMed and all major indexing services

- Maximum visibility for your research

Submit your manuscript at www.biomedcentral.com/submit
Biomed Central 